\title{
Qualitative Aspects in Nonlocal Dynamics
}

\author{
G. M. Coclite ${ }^{1}$ D S. Dipierro ${ }^{2} \cdot$ G. Fanizza ${ }^{3} \cdot$ F. Maddalena ${ }^{1} \cdot$ M. Romano ${ }^{1} \cdot$ E. Valdinoci $^{2}$
}

Received: 21 June 2021 / Accepted: 15 September 2021 / Published online: 15 October 2021

(c) The Author(s) 2021

\begin{abstract}
In this paper, we investigate, through numerical studies, the dynamical evolutions encoded in a linear one-dimensional nonlocal equation arising in peridynamics. The different propagation regimes ranging from the hyperbolic to the dispersive, induced by the nonlocal feature of the equation, are carefully analyzed. The study of an initial value Riemann-like problem suggests the formation of a singularity.
\end{abstract}

Keywords Fractional wave propagation · Dispersive equation · Nonlocal evolutions

JEL classification $49 \mathrm{~J} 45 \cdot 74 \mathrm{~K} 30 \cdot 74 \mathrm{~K} 35 \cdot 74 \mathrm{R} 10$

\section{Introduction}

In the previous works [1, 2], some relevant mathematical features characterizing the dynamical evolution ruled by a peridynamic model introduced by S.A. Silling ([3-7]) have been studied in detail by focusing on the mathematical consistency (see also [8]) of this new formulation of continuum mechanics. The nonlocal effects related to this model

G. M. Coclite

giuseppemaria.coclite@poliba.it

S. Dipierro

serena.dipierro@uwa.edu.au

G. Fanizza

gfanizza@fc.ul.pt

F. Maddalena

francesco.maddalena@poliba.it

M. Romano

marzia.romano@poliba.it

E. Valdinoci

enrico.valdinoci@uwa.edu.au

1 Dipartimento di Meccanica, Matematica e Management, Politecnico di Bari, Via E. Orabona 4, Bari I-70125, Italy

2 Department of Mathematics and Statistics, University of Western Australia, 35 Stirling Highway, Crawley WA6009, Australia

3 Instituto de Astrofisica e Ciencias do Espaco, Faculdade de Ciencias, Universidade de Lisboa, Edificio C8, Campo Grande P-1740-016, Lisbon, Portugal 
represent a very interesting problem in mathematical physics which has captured the interest of many researchers during the past decades (see, e.g., [9-15]).

In the present paper, we exploit a qualitative analysis of a one-dimensional linear peridynamical model to highlight the behavior of the solutions of an initial value problem in dependence of the characteristics affecting the nonlocal properties of the equation. More precisely, we show through numerical investigations the way nonlocality rules a wide range of modes of wave propagation (see also [16, 17]). Indeed, the dynamics predicted by the equation under study exhibits a behavior ranging from the hyperbolic-like to the dispersive-like propagation ([18]), according to its highly nontrivial dispersive relation. These new phenomena, unpredictable in classical continuum mechanics ([19]), are ruled by two constitutive parameters both related to the nonlocal character of the equation.

The paper is organized as follows. In Sect. 2, we introduce the main initial value problem in terms of a linear peridynamic model and the related dispersion relation focusing on the asymptotic limits. Moreover, the important notion of group velocity ruling the transport of energy is analyzed. In Sect. 3, we study the evolution of an initial localized profile in the range of the main parameters characterizing the problem. The shape of the initial datum is contrasted with the parameters affecting nonlocality, thus revealing an entire catalogue of different interesting behaviors. In Sect. 4, we show how a suitable choice of parameters delivers the case of purely hyperbolic propagation exhibiting traveling wave solution. Eventually, in Sect. 5 we explore the possible occurrence of singularities ([20]), allowed in principle for energy finite solutions. To this end, the study of a Riemann-like problem allows room for the formation of a singularity.

\section{One-Dimensional Peridynamic Model}

We perform a qualitative study regarding the phenomenology of the linear peridynamic model

$$
\begin{cases}\rho u_{t t}=-2 \kappa \int_{-\delta}^{\delta} \frac{u(t, x)-u(t, x-y)}{|y|^{1+2 \alpha}} d y=: K(u), & t>0, x \in \mathbb{R}, \\ u(0, x)=v_{0}(x), & x \in \mathbb{R}, \\ u_{t}(0, x)=v_{1}(x), & x \in \mathbb{R},\end{cases}
$$

for some given constants $\alpha \in(0,1), \kappa>0, \delta>0$.

As shown in [1], this problem admits the unique formal solution

$$
u(t, x)=\int_{\mathbb{R}} e^{-i \xi x}\left[\widehat{v_{0}}(\xi) \cos (\omega(\xi) t)+\frac{\widehat{v_{1}}(\xi)}{\omega(\xi)} \sin (\omega(\xi) t)\right] d \xi,
$$

where $\widehat{v_{0}}(\xi)$ and $\widehat{v_{1}}(\xi)$ are, respectively, the Fourier transforms of the initial conditions $v_{0}(x)$ and $v_{1}(x)$ and

$$
\omega(\xi):=\left(\frac{2 \kappa}{\rho \delta^{2 \alpha}} \int_{-1}^{1} \frac{1-\cos (\xi \delta z)}{|z|^{1+2 \alpha}} d z\right)^{1 / 2},
$$

is the dispersive relation actually governing the dynamics and $\xi$ is the wavenumber variable. Eq. (3) admits solution for a wide class of initial conditions (see [1] for the rigorous discussion in this regard). However, in this manuscript, we limit our phenomenological investigation to the particular case of initial conditions formally corresponding to a 
(possibly approximate) traveling wave. This case is described with any loss of generality by the constraint

$$
v_{1}(x)=-v v_{0}^{\prime}(x)
$$

where $v$ is a number denoting the initial velocity of the perturbations (or, in frequency space, one can recast Eq. (4) in the form $\widehat{v_{1}}(\xi)=i v \xi \widehat{v_{0}}(\xi)$ ). Eq. (4) can be easily understood as the limit for $t \rightarrow 0$ of the velocity of a traveling waveform $f(x-v t)$ : indeed, its velocity is just $\partial_{t} f(x-v t)=-v \partial_{q} f(q)$, where $q=x-v t$. Hence, in the above-mentioned limit, $q \rightarrow x$ and the constraint (4) follows.

The physical interest in the traveling wave initial conditions stands in the comparison with the linear 1D wave equation. Indeed, since the latter admits non-dispersive propagations, any traveling wave initial conditions will evolve as at most two independent traveling waves, according to the value of $v$. On the opposite, Eq. (3) exhibits a highly non-trivial relation between $\omega$ and $\xi$. More precisely, it has been proven in [1] that Eq. (3) admits two characteristic behaviors discriminated by the length scale $\delta$, namely

$$
\begin{aligned}
\text { if } \xi \ll 1 / \delta \text { then } \omega \sim \xi, \\
\text { if } \xi \gg 1 / \delta \text { then } \omega \sim \xi^{\alpha} .
\end{aligned}
$$

More precisely

$$
\begin{array}{r}
\lim _{\xi \rightarrow 0^{+}} \frac{\omega(\xi)}{\xi}=\delta^{1-\alpha} \sqrt{\frac{\kappa}{\rho(1-\alpha)}}, \\
\lim _{\xi \rightarrow \infty} \frac{\omega(\xi)}{\xi^{\alpha}}=\sqrt{\frac{4 \kappa}{\rho} \int_{0}^{\infty} \frac{1-\cos \tau}{\tau^{1+2 \alpha}} d \tau}=\sqrt{-\frac{4 \kappa}{\rho} \cos (\pi \alpha) \Gamma(-2 \alpha)},
\end{array}
$$

where $\Gamma$ is the Euler gamma function. Hence, for large scale modes, the dynamics is practically non-dispersive and these modes travel with group velocity $v_{g} \equiv \omega^{\prime}(\xi) \approx \delta^{1-\alpha} \sqrt{\frac{\kappa}{\rho(1-\alpha)}}$. On the other hand, small scale modes experience a sub-linear dispersive dynamics with group velocity $v_{g} \approx \xi^{\alpha-1} \sqrt{\frac{4 \kappa}{\rho} \int_{0}^{\infty} \frac{1-\cos \tau}{\tau^{1+2 \alpha}} d \tau}$. According to the initial conditions, qualitative differences with respect to the classic case are expected when small scales are switched on along the dynamics. In the following section, we will explore several scenarios to point out this peculiarity.

Let us remark that $\alpha \in(0,1)$ plays a crucial role in the subsequent analysis. Indeed, besides ruling the smoothness of the admissible solutions for the problem (1), it is responsible in tuning the effects of the nonlocal feature in the present theory. More precisely, the higher the $\alpha$, the more hyperbolic the propagation, whereas the lower the $\alpha$, the stronger the nonlocal effects, leading to dispersive propagation.

We conclude this section by proving for the Cauchy problem in (1) that the group velocity given by

$$
v_{g}:=\omega^{\prime}(\xi)
$$

represents (see [21]) the velocity of propagation of the energy density of monochromatic waves. To this end, we define the energy density $e$ 


$$
e:=\frac{\rho}{2}\left|u_{t}\right|^{2}+W
$$

and the energy flux $G$

$$
G:=\omega \partial_{\xi} W
$$

where $W$ is the potential energy density

$$
W:=\frac{\kappa}{2} \int_{-\delta}^{\delta} \frac{|u(t, x)-u(t, x-y)|^{2}}{|y|^{1+2 \alpha}} d y .
$$

In this setting, we consider a monochromatic wave

$$
u(t, x)=A e^{i \omega(\xi) t} e^{i \xi x}
$$

Here, for simplicity, up to a phase shift, we assume that $A \in \mathbb{R}$. At a formal level, this wave would correspond to picking a $\xi \in \mathbb{R}$ and choosing as initial conditions $v_{0}$ such that $\widehat{v_{0}}$ is the Dirac delta at $\xi$ and $v_{1}$ such that (4) is satisfied. We claim that

$$
v_{g}=\frac{G}{e} .
$$

To check this, we observe that the potential energy density of the monochromatic wave is given by

$$
\begin{aligned}
W & =\frac{\kappa}{2} \int_{-\delta}^{\delta} \frac{|u(t, x)-u(t, x-y)|^{2}}{|y|^{1+2 \alpha}} d y \\
& =\frac{\kappa A^{2}}{2} \int_{-\delta}^{\delta} \frac{\left|e^{i \xi x}-e^{i \xi(x-y)}\right|^{2}}{|y|^{1+2 \alpha}} d y \\
& =\frac{\kappa A^{2}}{2} \int_{-\delta}^{\delta} \frac{\left|1-e^{-i \xi y}\right|^{2}}{|y|^{1+2 \alpha}} d y \\
& =\frac{\kappa A^{2}}{2} \int_{-\delta}^{\delta} \frac{[1-\cos (\xi y)]^{2}+\sin ^{2}(\xi y)}{|y|^{1+2 \alpha}} d y \\
& =\kappa A^{2} \int_{-\delta}^{\delta} \frac{1-\cos (\xi y)}{|y|^{1+2 \alpha}} d y \\
& =\kappa A^{2} \delta^{-2 \alpha} \int_{-1}^{1} \frac{1-\cos (\xi \delta z)}{|z|^{1+2 \alpha}} d y \\
& =\frac{\rho A^{2}}{2} \omega^{2}(\xi) .
\end{aligned}
$$

On the other hand, the total energy density is given by

$$
e=\frac{\rho}{2}\left|u_{t}\right|^{2}+W=\rho A^{2} \omega^{2}(\xi)
$$

It then follows that

$$
\frac{G}{e}=\omega(\xi) \frac{\partial_{\xi} W}{e}=\frac{\omega^{2}(\xi) \rho A^{2} \omega^{\prime}(\xi)}{\rho A^{2} \omega^{2}(\xi)}=\omega^{\prime}(\xi) \equiv v_{g},
$$


hence, the relation in (7) is proven. This result is interesting since it extends the classical result for the wave equation $[18,21]$ to the linear peridynamic model.

\section{Localized Initial Conditions}

In this section, we study the dispersive phenomena of the linear peridynamic model in the range of parameters of $\alpha$ and $\delta$. As a first step, we fix our constitutive parameters as $\rho=1$ and $\kappa=1 / 2$ and leave them unchanged for the rest of the discussion. Aiming to study the traveling waves, we then consider Gaussian initial conditions

$$
v_{0}(x)=\sqrt{2 \pi} e^{-\frac{x^{2}}{2 \sigma^{2}}} \quad \text { and } \quad v_{1}(x)=-v v_{0}(x) .
$$

The interesting feature of those initial conditions relies in their localization close to the origin. Indeed, we will provide numerical evidences that these localized initial conditions remain localized as time evolves. This suggests that the evolution experiences almost finite propagation velocity. From now on, the key point to have in mind will be always the relative amplitude between the two parameters $\delta$ and group velocity $v_{g}$ characterizing the nonlocality of (1) and the two parameters $\sigma$ and $v$ involved in the initial conditions.

Figures from 1 to 12 have been obtained with the trapz function of numpy module available in Python. Trapezoidal integrations have been performed within the range $\xi \in[-1000,1000]$ and with width of $10^{-2}$ for each trapezoid. Given these specifics, if we define $f(\xi, x, t)$ as the integrand of Eq. (2), the numerical error is controlled by the upper bound $0.02 \times\left|f_{\xi \xi}(\xi, x, t)\right|$. However, we underline that this error estimation is not optimal.

The rest of the section is divided into three parts, devoted to the analysis of the interplay between the nonlocal range $\delta$ and the amplitude of the initial condition $\sigma$, since their ratio plays a crucial role in occurrence of dispersive effects in the wave propagation. More precisely, when $\sigma$ is much smaller than $\delta$, the solution develops high oscillations typical of the dispersive regime almost immediately, showing the deep difference between the peridynamics and the wave equation. When $\sigma$ is of the same order of $\delta$, we capture the strong transport effect in short times and the occurrence of dispersion on the long range. Finally, when $\sigma$ is much bigger than $\delta$, the dynamics experiences the (almost) total absence of dispersion and the occurrence only of hyperbolic propagation, typical of the wave equation.

\subsection{Case $\sigma \ll \delta$}

This subsection is devoted to the choice

$$
\sigma=10^{-1} \text { and } \delta=1 .
$$

In order to support our claim about the immediate occurrence of dispersive behavior, independently of the choice of $\alpha$, we show different cases regarding three possible choices of $\alpha$.

In Fig. 1, we consider

$$
\alpha=10^{-1} \text {. }
$$

In Fig. 2, we consider 

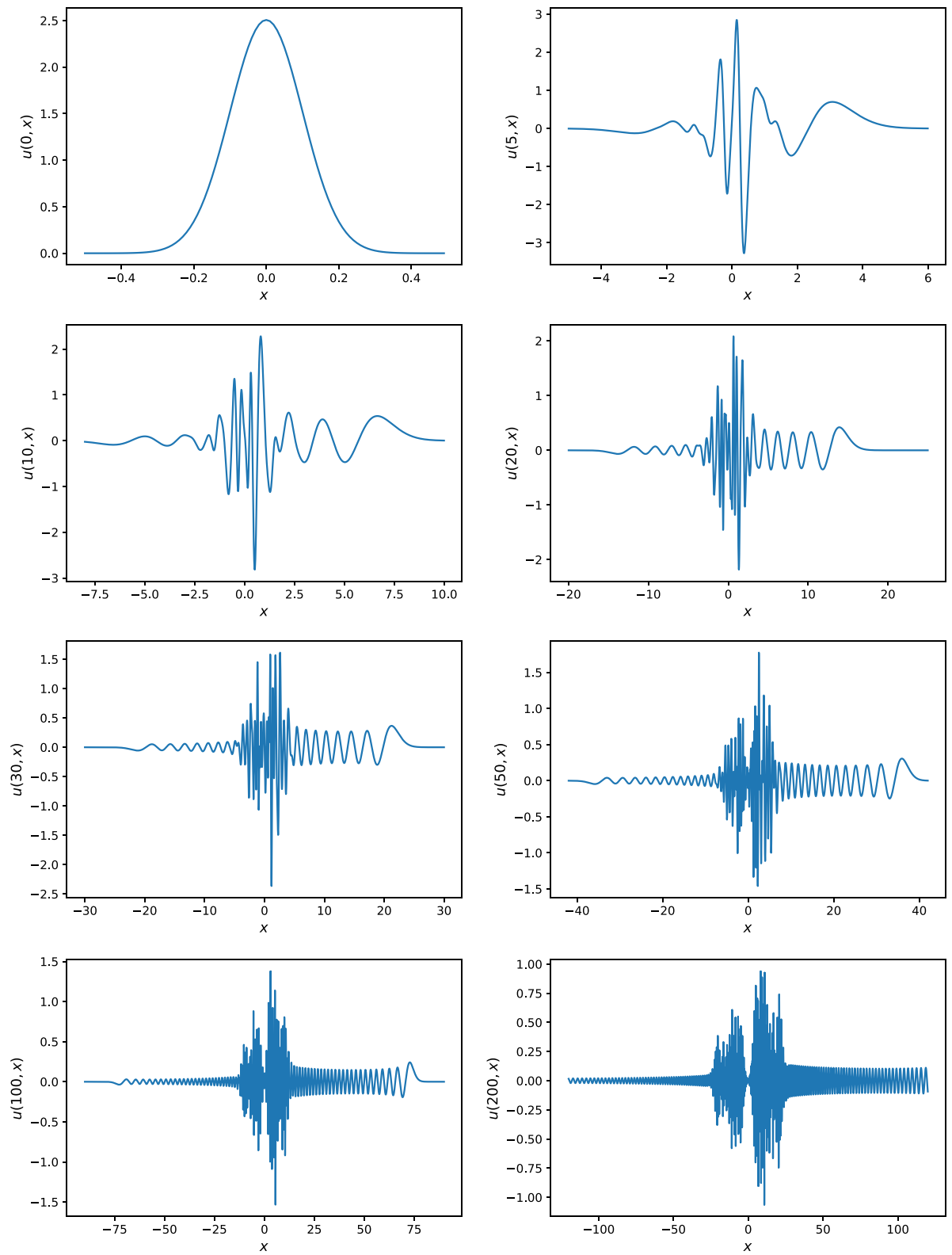

Fig. 1 Numerical simulations of (1)-(9) at different times in correspondence of $\sigma=10^{-1}<1=\delta, v=1$ and $\alpha=10^{-1}$

$$
\alpha=1 / 2 .
$$

In Fig. 3, we consider 

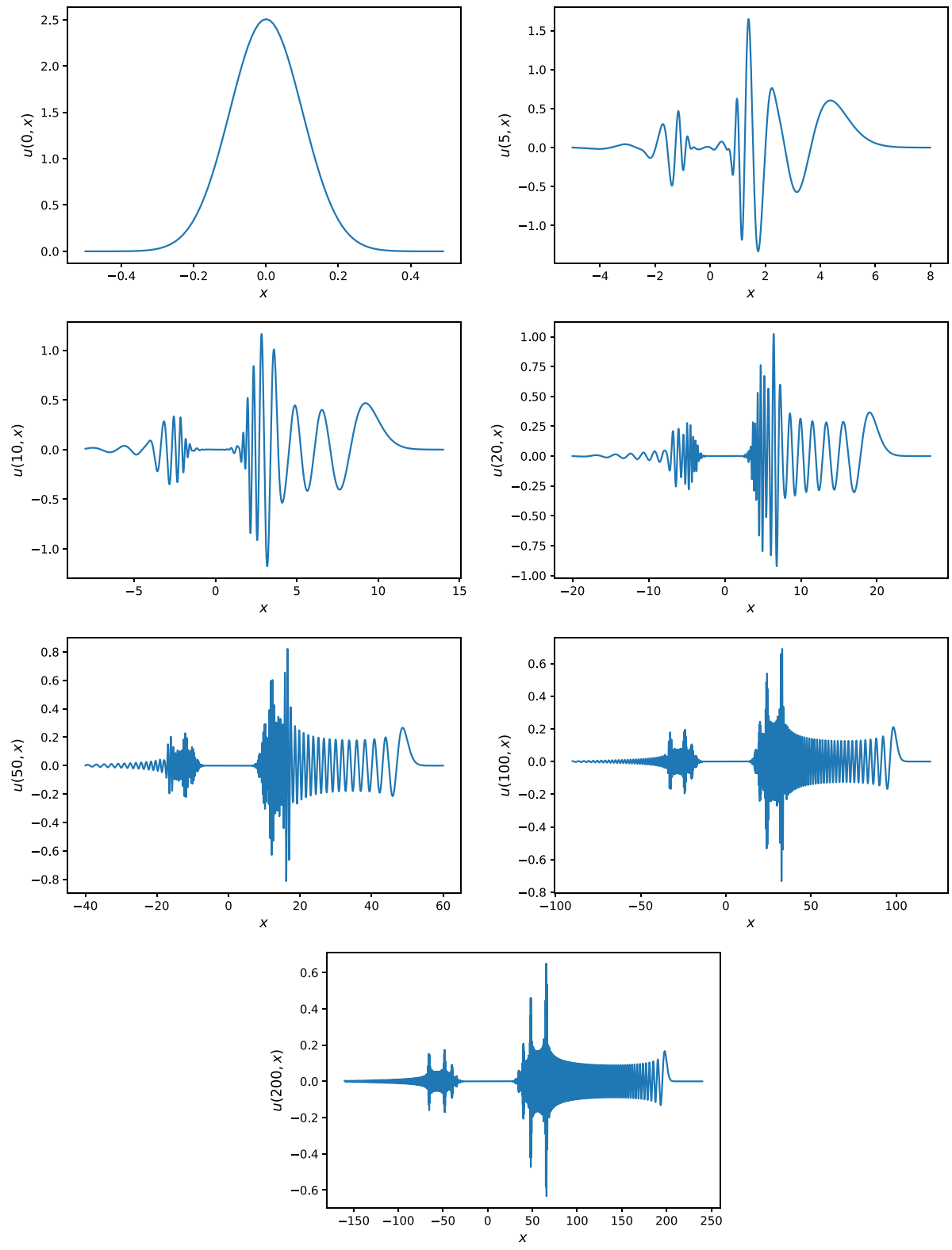

Fig. 2 Numerical simulations of (1)-(9) at different times in correspondence of $\sigma=10^{-1}<1=\delta, v=1$ and $\alpha=1 / 2$

$$
\alpha=9 / 10 .
$$



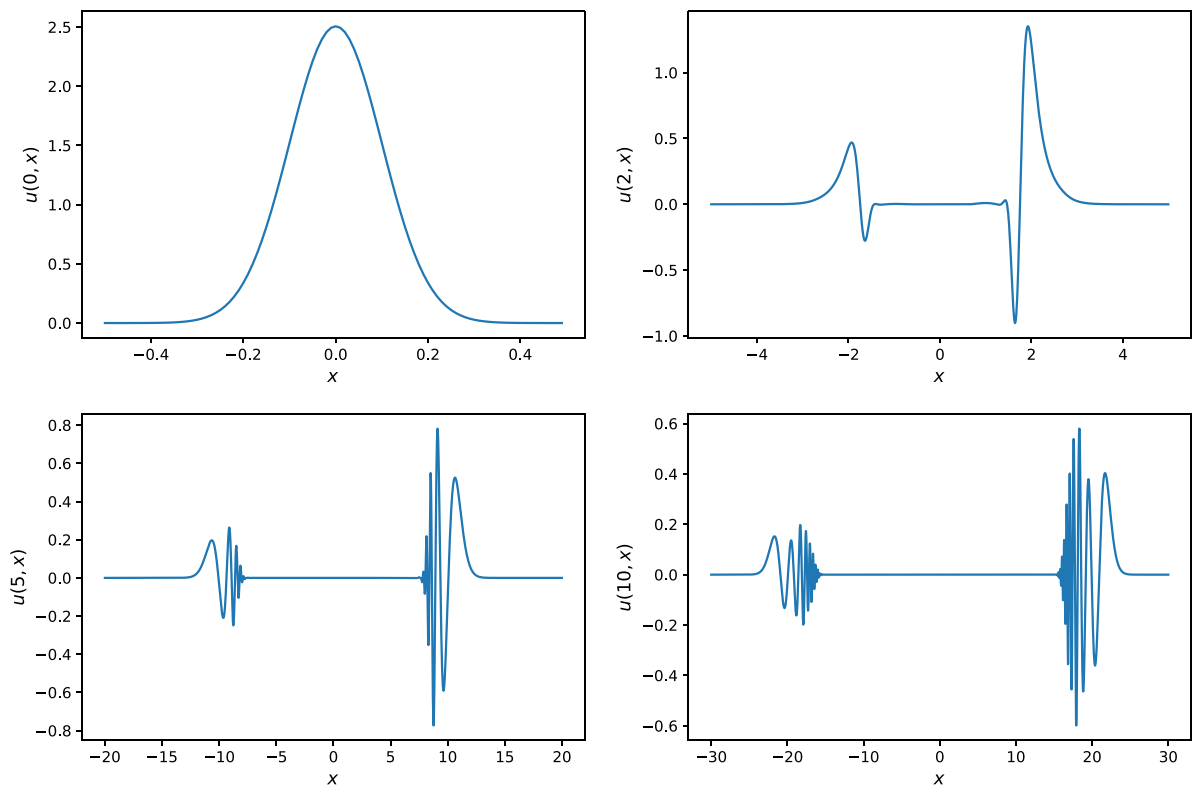

Fig. 3 Numerical simulations of (1)-(9) at different times in correspondence of $\sigma=10^{-1}<1=\delta, v=1$ and $\alpha=9 / 10$

\subsection{Case $\sigma \sim \delta$}

This subsection is devoted to the choice

$$
\sigma=1 \quad \text { and } \quad \delta=1 .
$$

In order to support our claim about the competitive occurrence of dispersive behavior and hyperbolic propagation, according of the value of $\alpha$, we show different cases regarding three possible choices of $\alpha$.

In Fig. 4, we consider

$$
\alpha=10^{-1}
$$

In Fig. 5, we consider

$$
\alpha=1 / 2
$$

In Fig. 6, we consider

$$
\alpha=9 / 10 \text {. }
$$



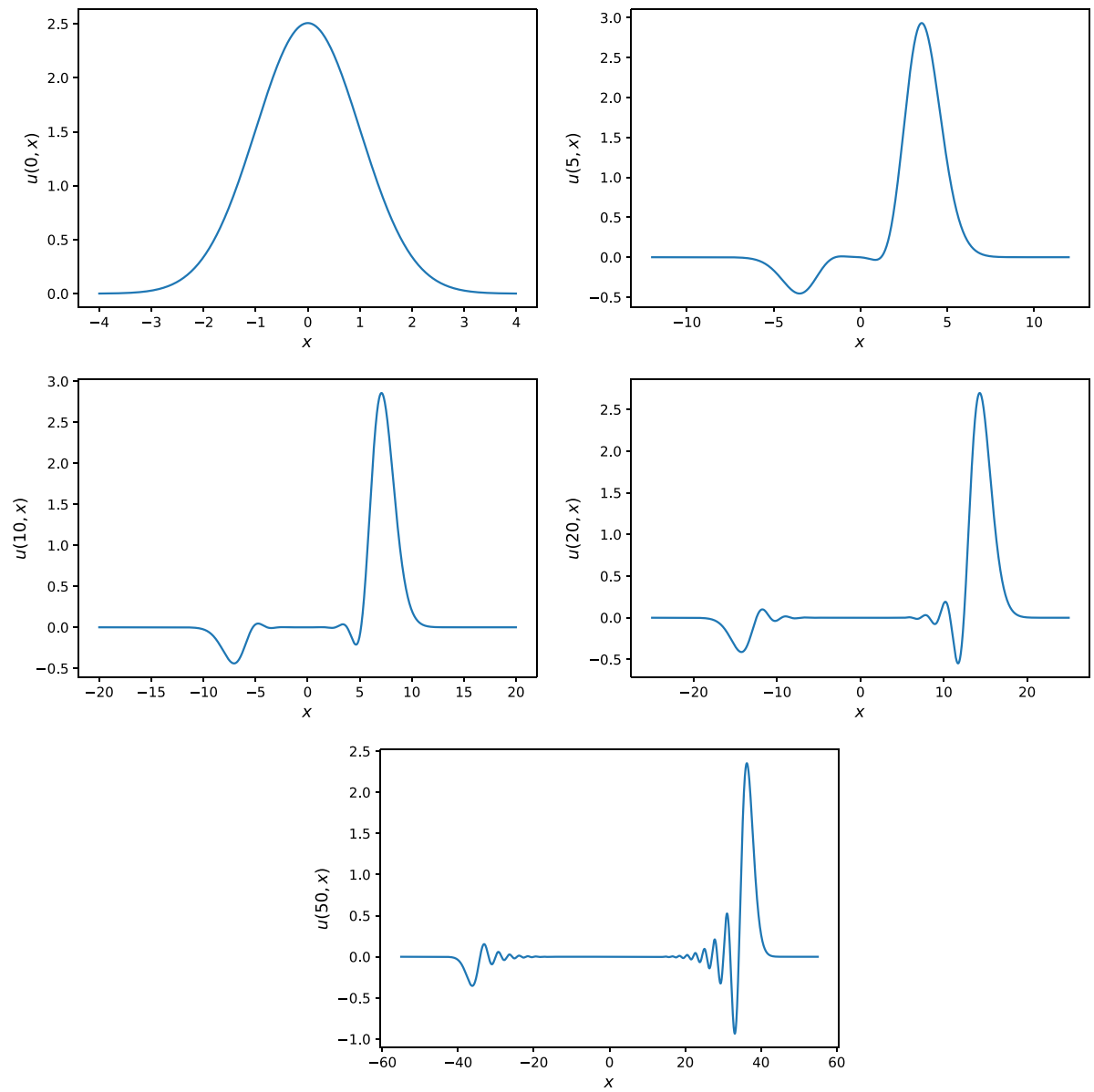

Fig. 4 Numerical simulations of (1)-(9) at different times in correspondence of $\sigma=\delta=1, v=1$ and $\alpha=10^{-1}$

\subsection{Case $\sigma \gg \delta$}

This subsection is devoted to the choice

$$
\sigma=10 \quad \text { and } \quad \delta=1 .
$$

In order to support our claim about the pure hyperbolic propagation and (almost) total absence of dispersive behavior, with respect to the value of $\alpha$, we show different cases regarding three possible choices of $\alpha$.

In Fig. 7, we consider

$$
\alpha=10^{-1} .
$$

In Fig. 8, we consider 

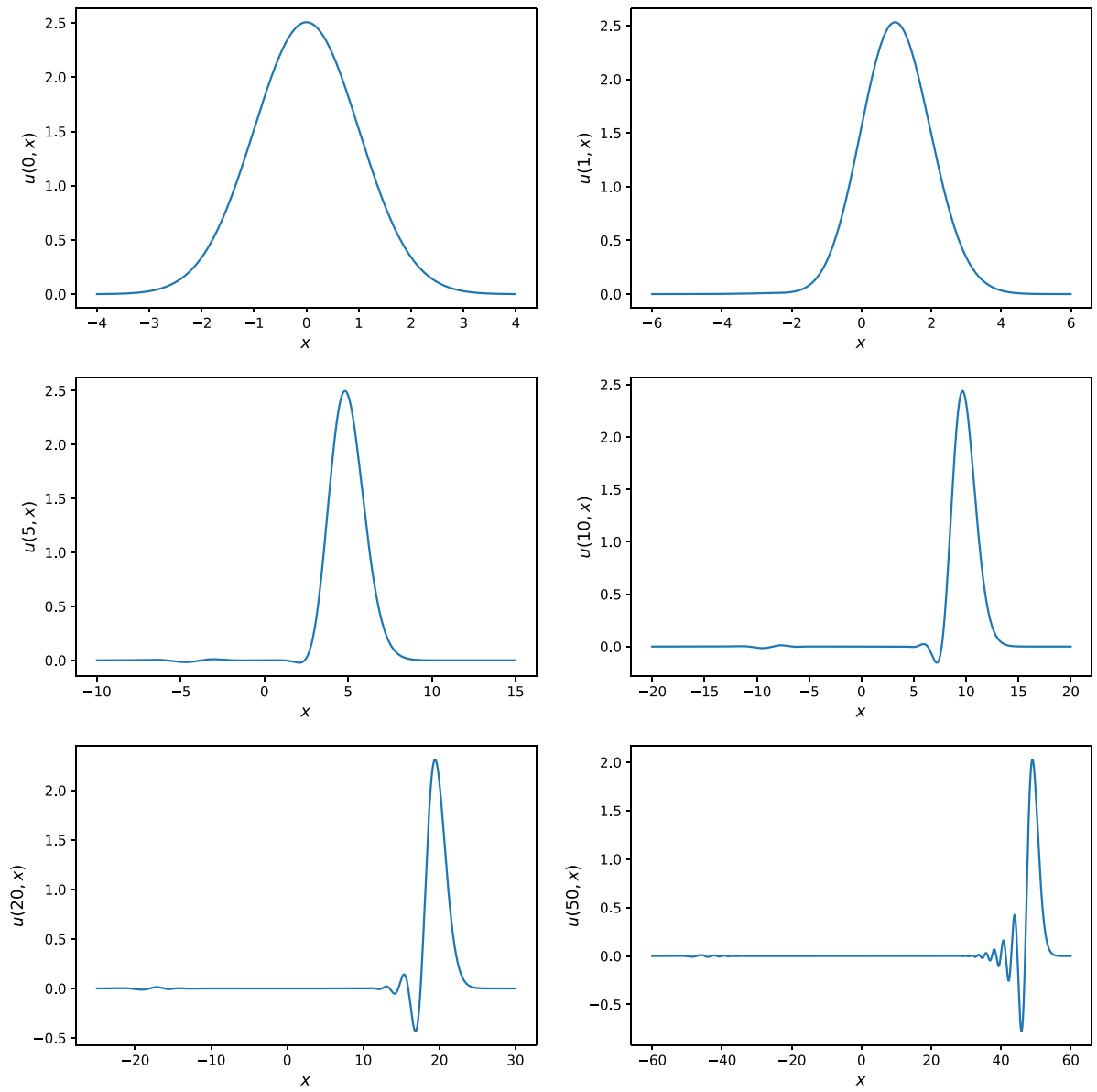

Fig. 5 Numerical simulations of (1)-(9) at different times in correspondence of $\sigma=\delta=1, v=1$ and $\alpha=1 / 2$

$$
\alpha=1 / 2 .
$$

In Fig. 9, we consider

$$
\alpha=9 / 10 .
$$

From the previous numerical analysis, we emphasize an important feature affecting the propagation problem under study. Indeed, all the previous plots clearly put in evidence the existence of a propagation velocity $v_{p}$ bounded by

$$
v_{p} \leq \frac{\delta^{1-\alpha}}{\sqrt{2(1-\alpha)}}=: v_{\max } .
$$



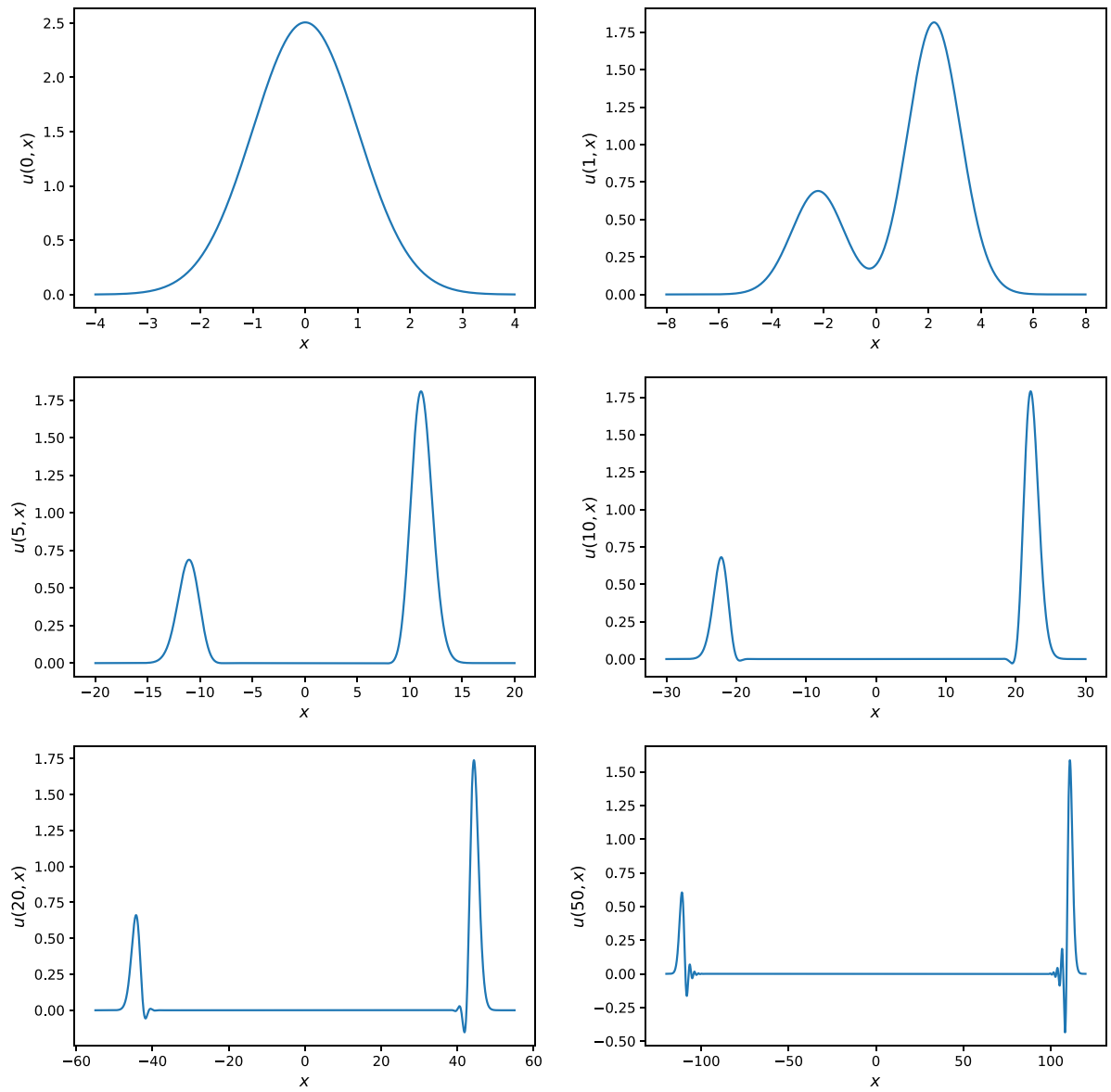

Fig. 6 Numerical simulations of (1)-(9) at different times in correspondence of $\sigma=\delta=1, v=1$ and $\alpha=9 / 10$

Heuristically, one can appreciate that for every $t$, the solutions live almost entirely within the region $|x| \leq v_{\max } t$. The physical reason behind the value of $v_{\max }$ stands in the fact that this is the upper bound of the group velocity $v_{g}$, namely no frequency can travel at a propagation velocity higher than $v_{\max }$ itself. In the next section, we will investigate in detail the finite propagation velocity ruling the purely hyperbolic case.

\section{Hyperbolic-Like Propagation}

This section is devoted to the special case when the solution is a (possibly approximated) traveling wave. For this case, we choose

$$
\sigma \gg \delta \quad \text { and } \quad v=\frac{\delta^{1-\alpha}}{\sqrt{2(1-\alpha)}} .
$$



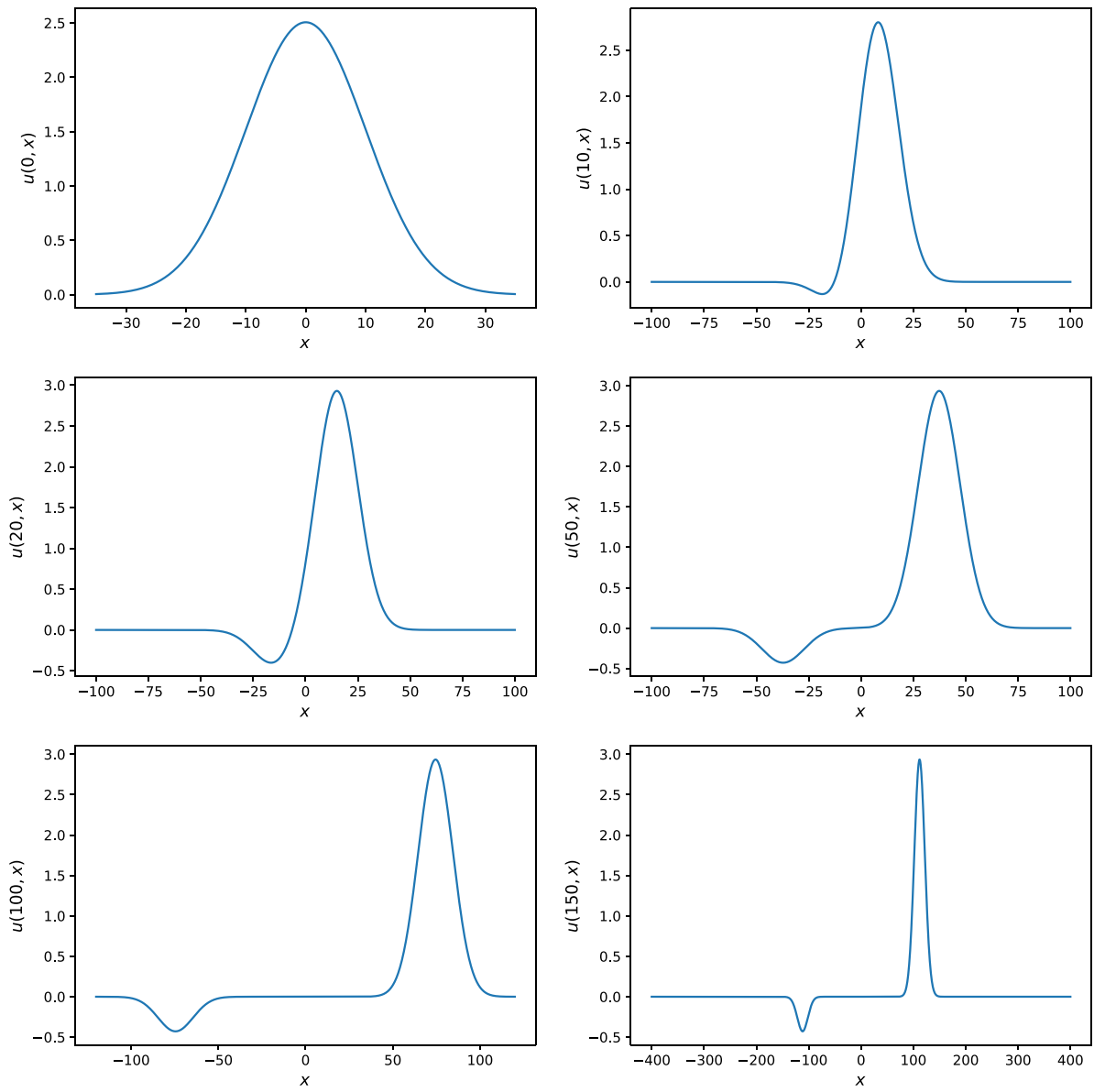

Fig. 7 Numerical simulations of (1)-(9) at different times in correspondence of $\sigma=10>1=\delta$, $v=1$ and $\alpha=10^{-1}$

In particular, we study the case

$$
\sigma=10 \text { and } \delta=1 .
$$

In Fig. 10, we consider

$$
\alpha=10^{-1} \text {. }
$$

In Fig. 11, we consider

$$
\alpha=1 / 2 .
$$

In Fig. 12, we consider

$$
\alpha=9 / 10 \text {. }
$$



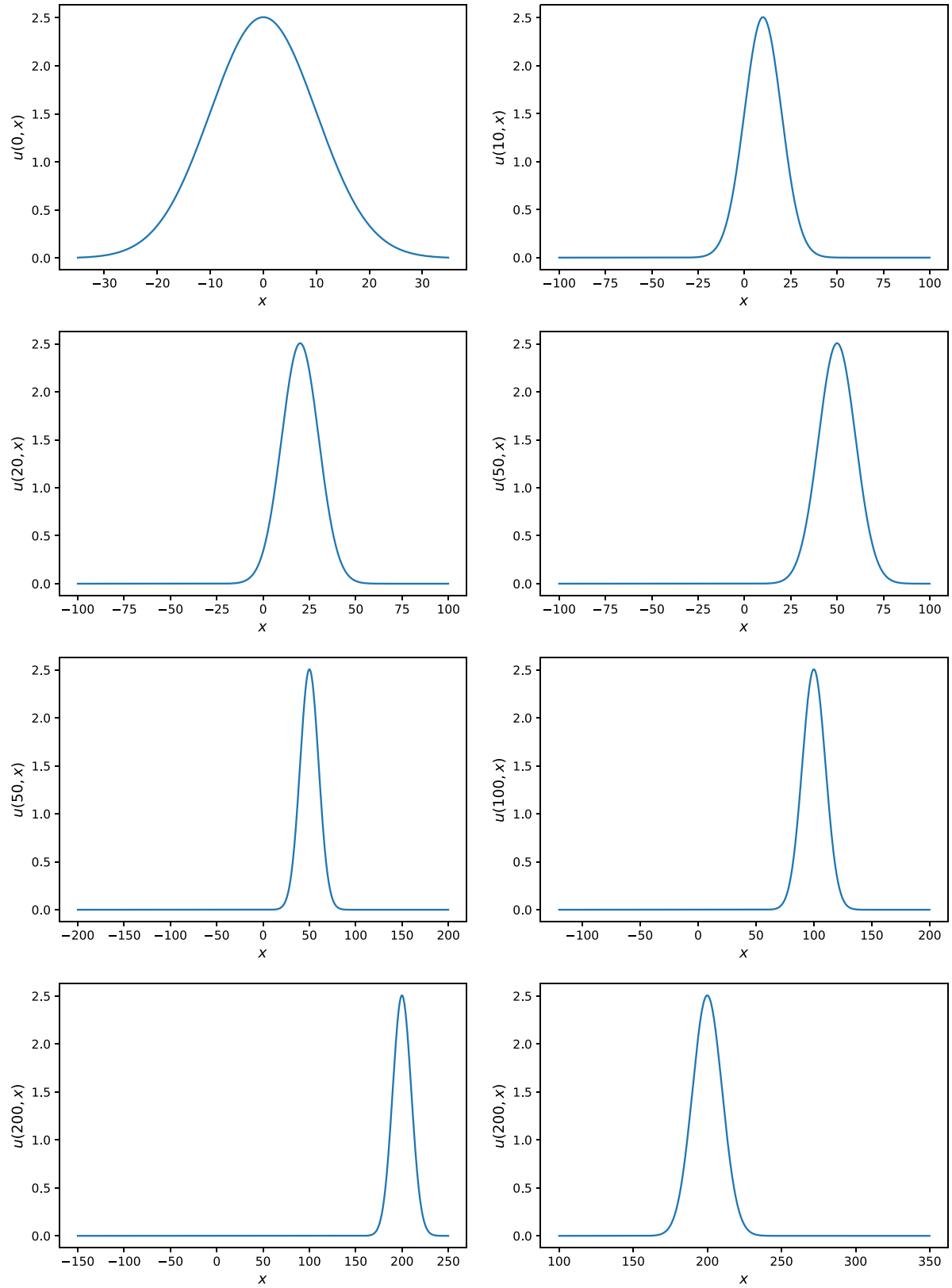

Fig. 8 Numerical simulations of (1)-(9) at different times in correspondence of $\sigma=10>1=\delta, v=1$ and $\alpha=1 / 2$ 

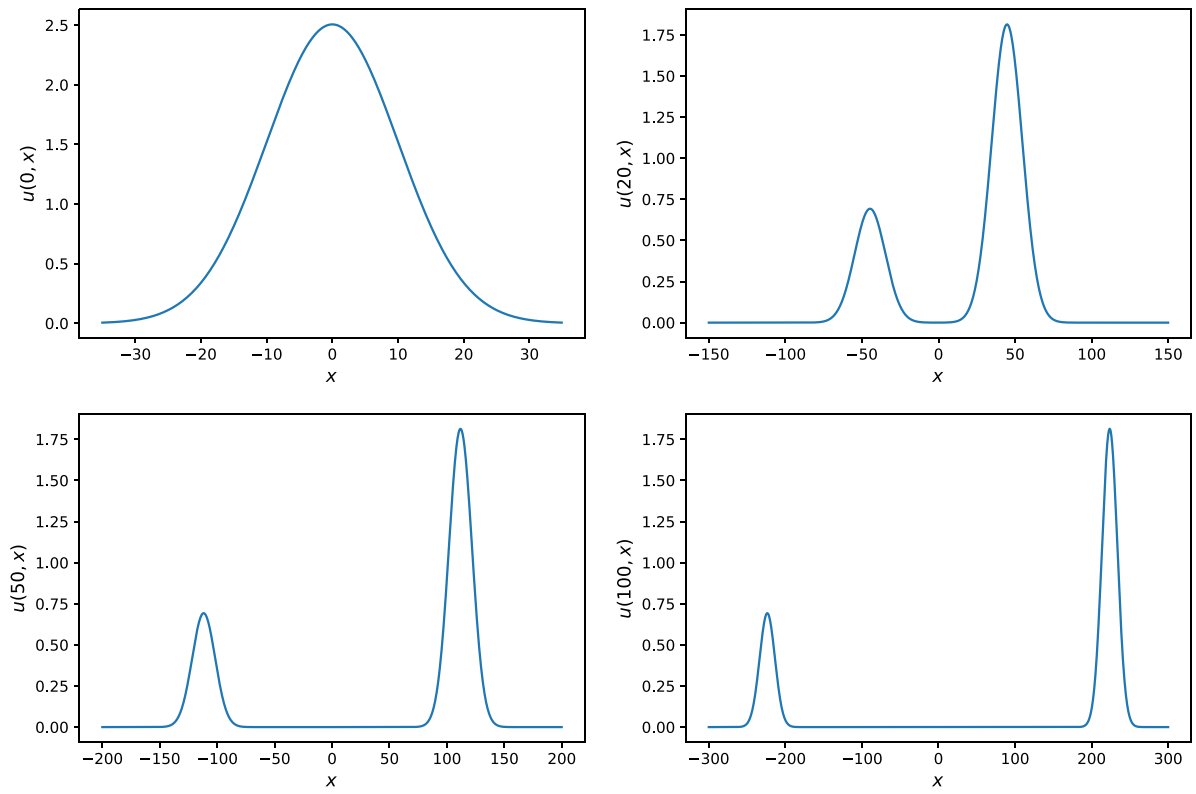

Fig. 9 Numerical simulations of (1)-(9) at different times in correspondence of $\sigma=10>1=\delta, v=1$ and $\alpha=9 / 10$
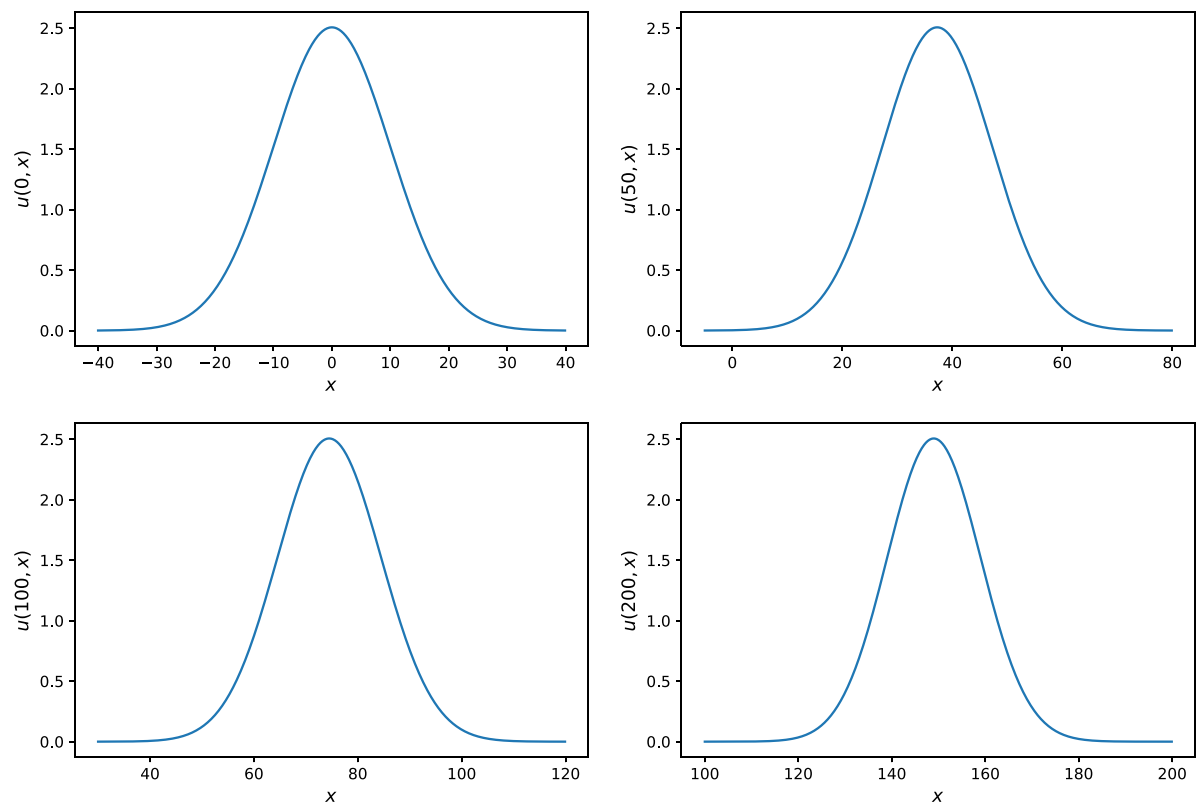

Fig. 10 Numerical solution at different times. We imposed Gaussian initial conditions with $\sigma=10 \delta$ and initial velocity $v=\delta^{1-\alpha} / \sqrt{2(1-\alpha)}$. This case refers to the parameters $\alpha=1 / 10$ and $\delta=1$ 

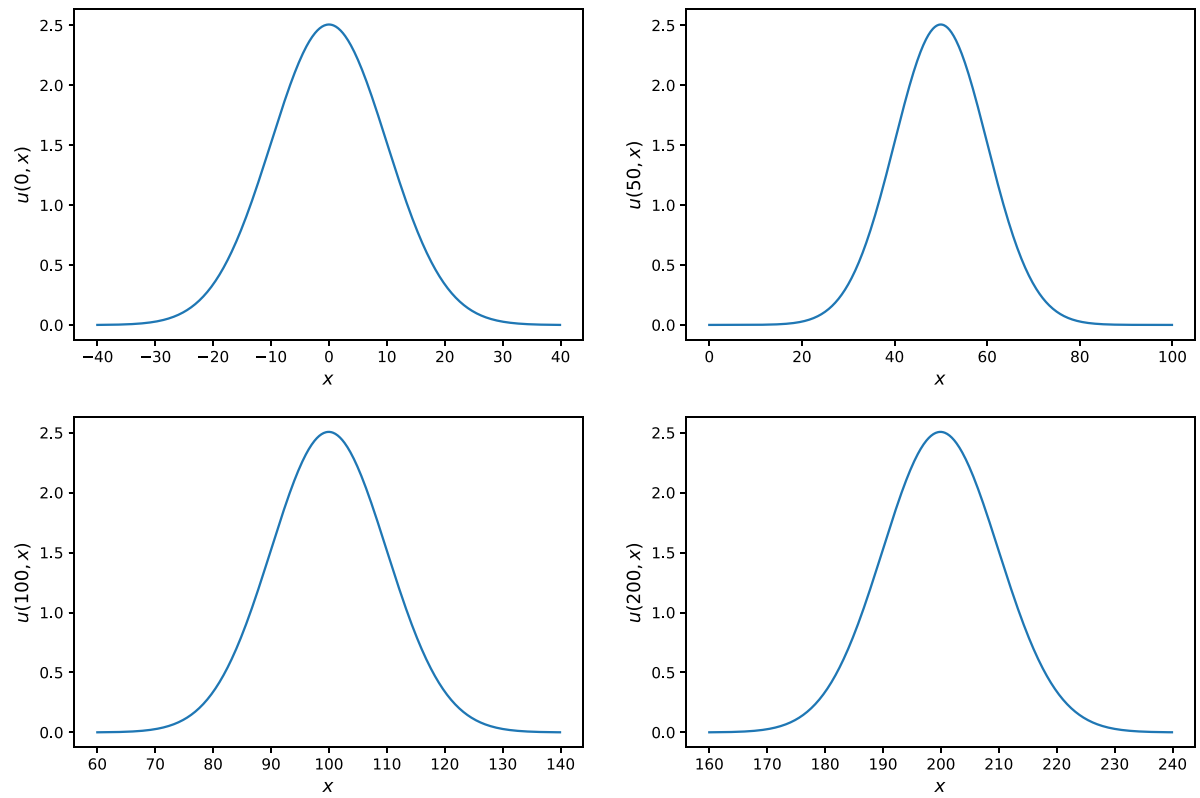

Fig. 11 Numerical solution at different times. We imposed Gaussian initial conditions with $\sigma=10 \delta$ and initial velocity $v=\delta^{1-\alpha} / \sqrt{2(1-\alpha)}$. This case refers to the parameters $\alpha=1 / 2$ and $\delta=1$
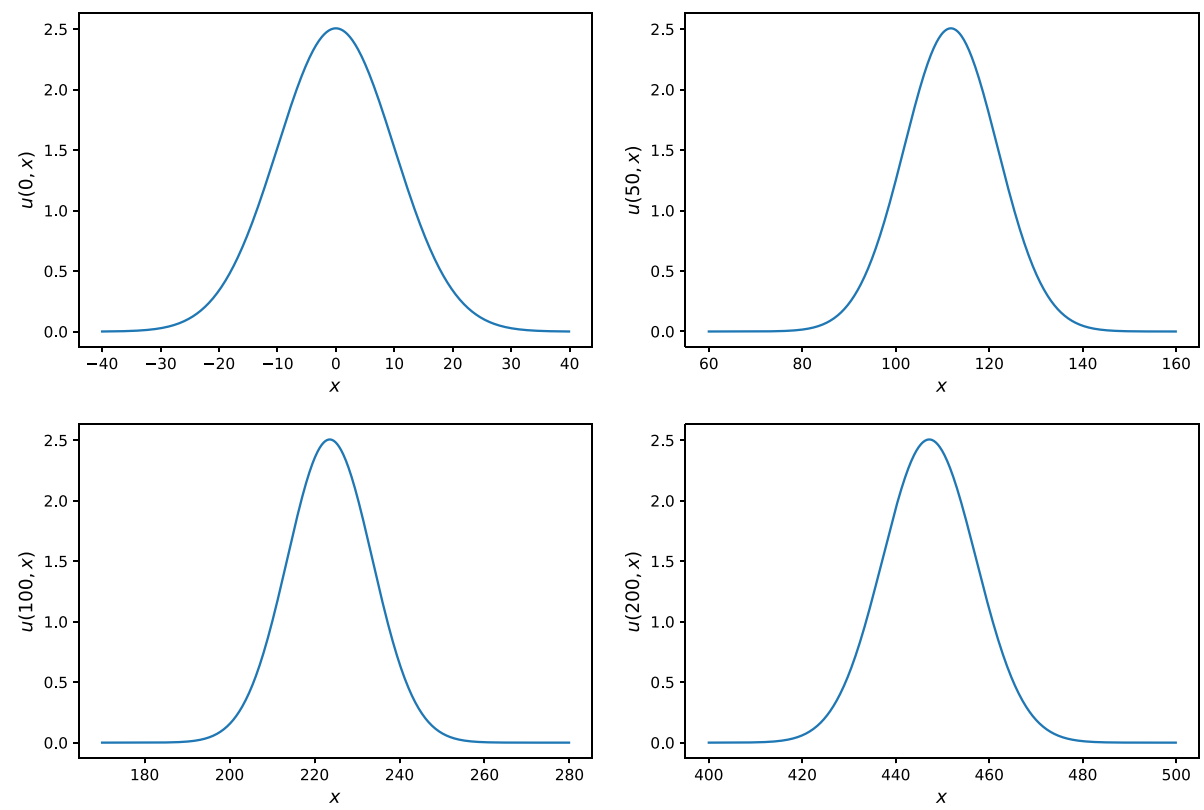

Fig. 12 Numerical solution at different times. We imposed Gaussian initial conditions with $\sigma=10 \delta$ and initial velocity $v=\delta^{1-\alpha} / \sqrt{2(1-\alpha)}$. This case refers to the parameters $\alpha=9 / 10$ and $\delta=1$ 

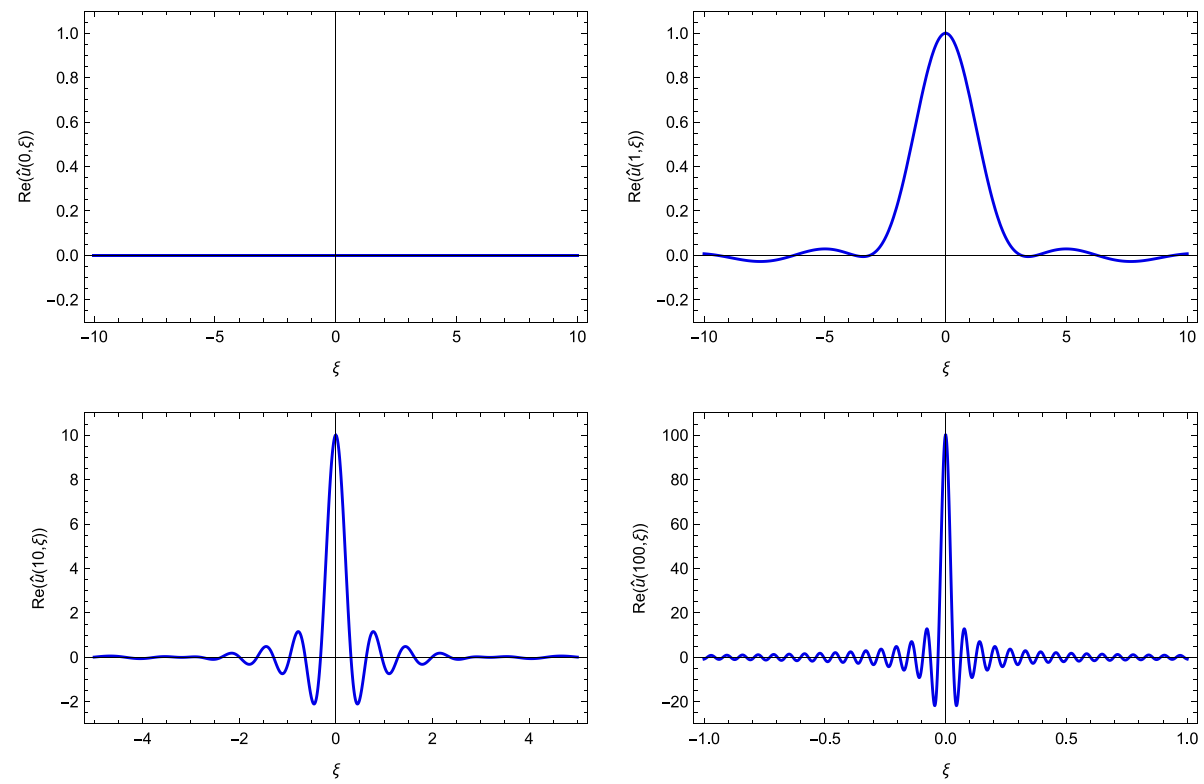

Fig. 13 Real part of the Fourier transform of the solution with initial conditions (12) at different times. This case refers to the parameters $\alpha=1 / 2$ and $\delta=1$

\section{Singularity Formation}

In this section, we explore the possible spontaneous creation of singularities. Following the analysis performed in $[22,23]$, one can try to study the following Riemann-like problem augmenting (1) with the following initial conditions

$$
v_{0}(x)=0 \quad \text { and } \quad v_{1}(x)=\left\{\begin{array}{lll}
v_{+} & \text {if } & x \geq 0 \\
v_{-} & \text {if } & x<0 .
\end{array}\right.
$$

Due to the fact that the Fourier transform of $v_{1}$

$$
\widehat{v_{1}}(\xi)=v_{+}\left(\pi \delta_{0}(\xi)+\frac{1}{i \xi}\right)+v_{-}\left(\pi \delta_{0}(-\xi)-\frac{1}{i \xi}\right)
$$

exhibits a strong singularity at $\xi=0$, rather than using the representation formula (2) in this case, we confine our analysis to some numerical experiments. For this, we truncate (11) and choose $v_{-}=0$ and $v_{+}=1$, namely

$$
v_{0}(x)=0 \quad \text { and } \quad v_{1}(x)=\chi_{[0,1]}(x) .
$$

Now, we have

$$
\widehat{v_{1}}(\xi)=\frac{i}{\xi}\left(1-e^{i \xi}\right),
$$

which does not exhibit the Dirac delta at $\xi=0$. 

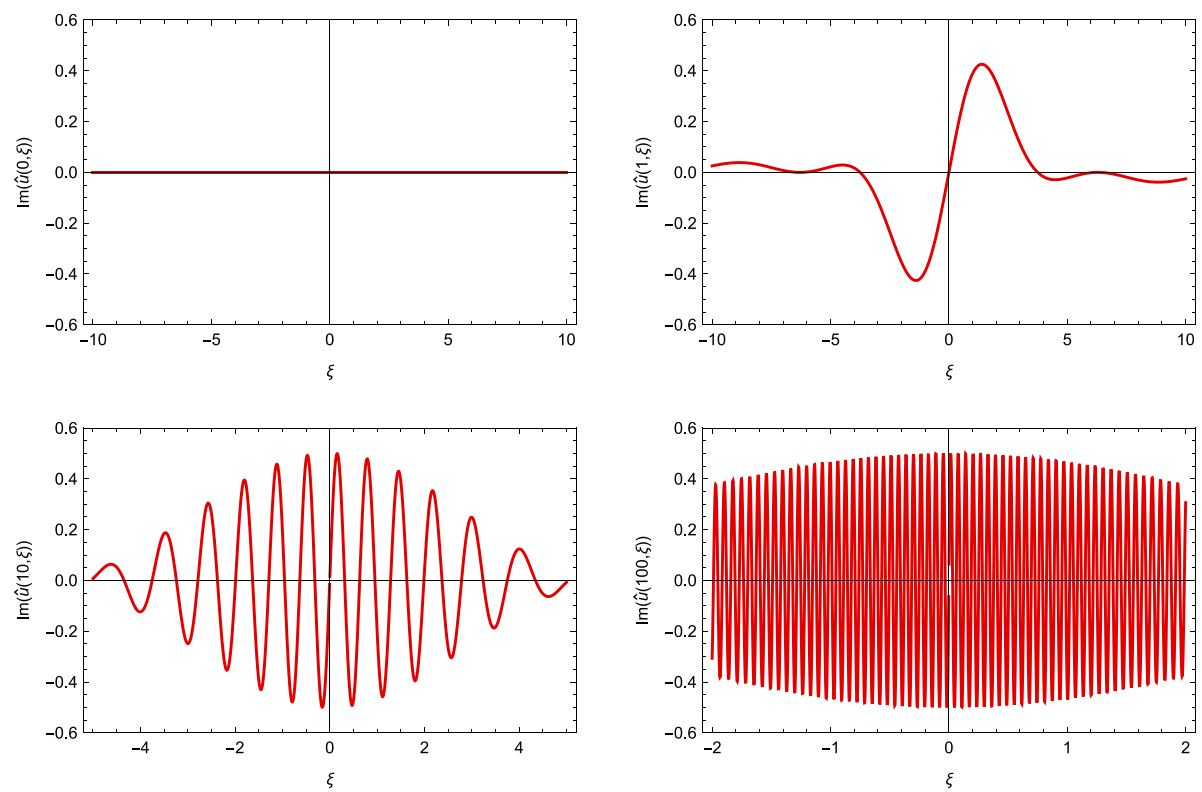

Fig. 14 Imaginary part of the Fourier transform of the solution with initial conditions (12) at different times. This case refers to the parameters $\alpha=1 / 2$ and $\delta=1$

In Figs. 13 and 14, we plot the real and imaginary parts of the Fourier transform of the solution expressed in (2) with initial data given in (12). We can appreciate that as time evolves the real part develops a peak at $\xi=0$, whereas the imaginary one becomes highly oscillating. Inspired by the analysis carried out in [23], we observe numerically a possible development of a stationary singularity at $x=0$.

\section{Conclusion}

In this paper, we have explored the features of the peridynamic model proposed in $[1,2]$ as a viable generalization of the wave equation for the study of nonlocal phenomena in the mechanics of continuous media. We have focused our phenomenological analysis to the 1D linear scenario. Despite its simplicity, this case study already exhibits deep differences with respect to the standard wave equation. In particular, dispersive propagation can occur when small scales dynamics is non-negligible.

This competitive behavior between classical hyperbolic propagation and dispersive phenomena is intimately related to the multiscale essence of the peridynamic framework and the crossover from one regime to the other one is dictated by the interaction length $\delta$ and the interaction scaling $\alpha$. Moreover, we have also shown that the energy propagates with the group velocity, regardless of the scale lengths involved in the dynamics.

From these general features, we have then exploited the phenomenology of the case of Gaussian initial condition with non-trivial velocity. In this scenario, our numerical investigation has shown that dispersive phenomena are relevant when the width $\sigma$ of the Gaussian initial profile is smaller than the peridynamic radius $\delta$. On the contrary, when $\sigma$ is much 
greater than $\delta$, our numerical investigation exhibits a hyperbolic-like propagation. This regime can be interpreted as a direct consequence of the multiscale nature of the dispersive relation $\omega(\xi)$. This is nearly linear when $\xi \ll \delta^{-1}$ and grows with a sub-linear rate when $\xi \gg \delta^{-1}$.

As a final comparison between the hyperbolic and dispersive behaviors, for the case $\sigma \gg \delta$, we have shown the existence of a typical initial velocity of the Gaussian initial condition leading to traveling wave-like solutions. Once again, the cornerstone to understand this behavior is the dispersive relation $\omega(\xi)$ : indeed this fine-tuned choice for the initial velocity is given by the limit for $\xi \rightarrow 0$ of the group velocity $\omega^{\prime}(\xi)$, which is a constant, as a consequence of the linearity of $\omega(\xi)$ on large scale. This limit is also responsible for the almost finite propagation of the solutions over time.

Finally, in the last section, we have discussed the possible spontaneous formation of singularities developed in an initial-value problem with discontinuous velocity datum.

Funding Open access funding provided by Politecnico di Bari within the CRUI-CARE Agreement.

\section{Declarations}

Conflicts of Interest The authors declare that they do not have any conflict of interest.

Open Access This article is licensed under a Creative Commons Attribution 4.0 International License, which permits use, sharing, adaptation, distribution and reproduction in any medium or format, as long as you give appropriate credit to the original author(s) and the source, provide a link to the Creative Commons licence, and indicate if changes were made. The images or other third party material in this article are included in the article's Creative Commons licence, unless indicated otherwise in a credit line to the material. If material is not included in the article's Creative Commons licence and your intended use is not permitted by statutory regulation or exceeds the permitted use, you will need to obtain permission directly from the copyright holder. To view a copy of this licence, visit http://creativecommons.org/licenses/by/4.0/.

\section{References}

1. Coclite GM, Dipierro S, Fanizza G, Maddalena F, Valdinoci E (2021) Dispersive effects in a peridynamic model

2. Coclite GM, Dipierro S, Maddalena F, Valdinoci E (2018) Wellposedness of a nonlinear peridynamic model. Nonlinearity 32(1):1-21

3. Silling S, Lehoucq R (2010) Peridynamic theory of solid mechanics. In H. Aref and E. van der Giessen, editors, Advances in Applied Mechanics, volume 44 of Advances in Applied Mechanics, pages 73-168. Elsevier

4. Silling SA (2000) Reformulation of elasticity theory for discontinuities and long-range forces. J Mech Phys Solids 48(1):175-209

5. Silling SA (2010) Linearized theory of peridynamic states. J Elast 99(1):85-111

6. Silling SA, Epton M, Weckner O, Xu J, Askari E (2007) Peridynamic states and constitutive modeling. J Elast 88(2):151-184

7. Silling SA, Lehoucq RB (2008) Convergence of peridynamics to classical elasticity theory. J Elast 93(1):13-37

8. Emmrich E, Puhst D (2015) Survey of existence results in nonlinear peridynamics in comparison with local elastodynamics. Comput Methods Appl Math 15(4):483-496

9. Eringen AC (2002) Nonlocal continuum field theories. Springer-Verlag, New York

10. Eringen AC, Edelen DGB (1972) On nonlocal elasticity. Int J Eng Sci 10:233-248

11. Kröner E (1967) Elasticity theory of materials with long range cohesive forces. Int J Solids Struct 3(5):731-742

12. Kunin IA (1982) Elastic media with microstructure. I, volume 26 of Springer Series in Solid-State Sciences. Springer-Verlag, Berlin-New York. One-dimensional models, Translated from the Russian 
13. Lim C, Zhang G, Reddy J (2015) A higher-order nonlocal elasticity and strain gradient theory and its applications in wave propagation. J Mech Phys Solids 78:298-313

14. Singh A, Das S, Altenbach H, Craciun EM (2020) Semi-infinite moving crack in an orthotropic strip sandwiched between two identical half planes. ZAMM Z Angew Math Mech 100(2):e201900202

15. Singh A, Das S, Craciun EM (2019) Effect of Thermomechanical Loading on an Edge Crack of Finite Length in an Infinite Orthotropic Strip. Mech Compos Mater 55(3):285-296

16. Coclite GM, Fanizzi A, Lopez L, Maddalena F, Pellegrino SF (2020) Numerical methods for the nonlocal wave equation of the peridynamics. Appl Numer Math 155:119-139

17. Lopez L, Pellegrino SF (2021) A spectral method with volume penalization for a nonlinear peridynamic model. Int J Num Methods Eng 122(3):707-725

18. Whitham GB (19996) Linear and nonlinear waves. Pure and Applied Mathematics (New York). John Wiley \& Sons, Inc., New York. Reprint of the 1974 original, A Wiley-Interscience Publication

19. Gurtin ME (1973) The linear theory of elasticity. In Linear theories of elasticity and thermoelasticity, pages 1-295. Springer

20. Coclite GM, Dipierro S, Maddalena F, Valdinoci E (2020) Singularity formation in fractional Burgers' equations. J Nonlinear Sci 30(4):1285-1305

21. Biot MA (1957) General theorems on the equivalence of group velocity and energy transport. Phys Rev 105:1129-1137

22. Wang L, Abeyaratne R (2018) A one-dimensional peridynamic model of defect propagation and its relation to certain other continuum models. J Mech Phys Solids 116:334-349

23. Weckner O, Abeyaratne R (2005) The effect of long-range forces on the dynamics of a bar. J Mech Phys Solids 53(3):705-728 